\title{
Indicações em transplante de células-tronco hematopoéticas em pacientes adultos com leucemia linfoide aguda
}

\author{
Indications for hematopoietic stem cell transplantation in adults in acute lymphoblastic leukemia
}

Maria Aparecida Zanichelli ${ }^{1}$

Vergilio R. Colturato ${ }^{2}$

Jairo Sobrinho
Na leucemia linfoide aguda (LLA), a proliferação, acúmulo e infiltração de células imaturas caracterizam uma entidade heterogênea, apresentando ampla diversidade de aspectos clínicos e biológicos. Na LLA do adulto, a concentração de fatores prognósticos de alto risco, como o imunofenótipo $B$, alterações cromossômicas e, principalmente, a presença do cromossomo $\mathrm{Ph}$ positivo. Considerações a respeito da alta morbidade e mortalidade relacionadas ao transplante de células-tronco hematopoéticas (TCTH) têm gerado controvérsias quanto à indicação desta modalidade terapêutica, nos pacientes adultos com LLA em primeira remissão $\left(1^{a} R C\right)$. Os resultados da terapia convencional com quimioterapia, diante dos diferentes grupos de risco em pacientes com LLA, têm sido utilizados para a indicação de TCTH. Apresentamos o algoritmo de indicações do transplante de células-tronco hematopoéticas em pacientes adultos com LLA. Rev. Bras. Hematol. Hemoter. 2010;32(Supl. 1):54-60.

Palavras-chave: Leucemia linfoide aguda; adultos; fatores de prognóstico; transplante de células-tronco hematopoéticas.

\section{Introdução}

A leucemia linfoide aguda (LLA), neoplasia hematológica que determina proliferação, acúmulo e infiltração de células imaturas, caracteriza-se por ser heterogênea, apresentando ampla diversidade de aspectos clínicos e biológicos.

$\mathrm{Na}$ infância, a LLA representa $80 \%$ das leucemias agudas, tendo perspectiva de cura de $80 \%$ diante do tratamento quimioterápico intensivo; já nos adultos, ela representa $20 \%$ das leucemias agudas com sobrevida global prolongada em torno de $30 \%$ a $40 \%$.

A partir de 15 anos de idade, existe mudança progressiva e significativa no comportamento biológico, agora desfavorável, nos pacientes portadores de LLA. Contribui de maneira expressiva para este cenário a grande concentração de fatores prognósticos negativos verificada na população adulta, por exemplo: a incidência de LLA -Ph1+é de $25 \%$ em adultos e $3 \%$ em crianças, ou a $t(12 ; 21)$ e a hiperdiploidia $>$ que 50 cromossomos, que, em crianças, constituem grupos de prognóstico favorável, totalizando $50 \%$ dos casos, não ocorrem nem em $10 \%$ da população adulta e, o pior, perdem o seu valor preditivo, favorável nesses pacientes.

$\mathrm{Na}$ atualidade são considerados fatores prognósticos negativos:

- Necessidade de um período superior a 4 semanas para a obtenção da remissão (falha indutória)

- Idade $>35$ anos

Alterações citogenéticas: $\mathrm{t}(9 ; 22) \mathrm{Ph}+, \mathrm{t}(4 ; 11)$, cariótipo complexo ( 5 ou mais anormalidades cromossômicas), hipodiploidia $(<40)$, triploidia

- DRM + após a indução

- Leucometria $>30.000 \mathrm{~mm}^{3}$ (linhagem B)

- Leucometria $>100.000 \mathrm{~mm}^{3}$ (linhagem T)

Considerações a respeito da alta morbidade e mortalidade relacionadas ao transplante de células-tronco hemato-

\footnotetext{
${ }_{2}^{l}$ Hematologista, Serviço de Hematologia e TMO, Hospital Brigadeiro - São Paulo-SP.

${ }^{2}$ Hematologista, Unidade de TMO do Hospital Amaral de Carvalho - Jaú-SP.

${ }^{3}$ Hematologista, Hospital Israelita Albert Einstein - São Paulo-SP.
}

Correspondência: Maria Aparecida Zanichelli

Av. Brigadeiro Luis Antonio $n^{\circ} 2651$

01401-901 - São Paulo-SP - Brasil

Email: zanichellima@uol.com.br - hemato.brigadeiro@uol.com.br

Doi: 10.1590/S1516-84842010005000030 
poéticas (TCTH) têm gerado controvérsias quanto à indicação desta modalidade terapêutica, nos pacientes adultos, com LLA em $1^{\text {a }}$ remissão $\left(1^{\mathrm{a}} \mathrm{RC}\right)$. De uma maneira geral, os resultados da terapia convencional com quimioterapia, diante dos diferentes grupos de risco em pacientes com LLA, têm sido utilizados para a indicação de TCTH.

Em 1994, o grupo LALA Sebban ${ }^{1}$ relatou que existia benefício em realizar o transplante em $1^{\mathrm{a}} \mathrm{RC}$ para os pacientes de alto risco e não para os pacientes de risco básico. Durante um bom período, esta conduta foi aceita nos diversos centros de transplante.

Em 2006, Gökbuget, Hoelzer² sugeriram a realização de TCTH em $1^{\text {a }}$ RC com doadores aparentados e/ou não aparentados, nos pacientes com LLA, com chance menor que $40 \%$ de sobrevida, diante da quimioterapia convencional.

Os dados dos registros internacionais indicam a LLA como sendo a segunda neoplasia com o maior número de procedimentos alogênicos (Figuras 1 e 2) e com maior

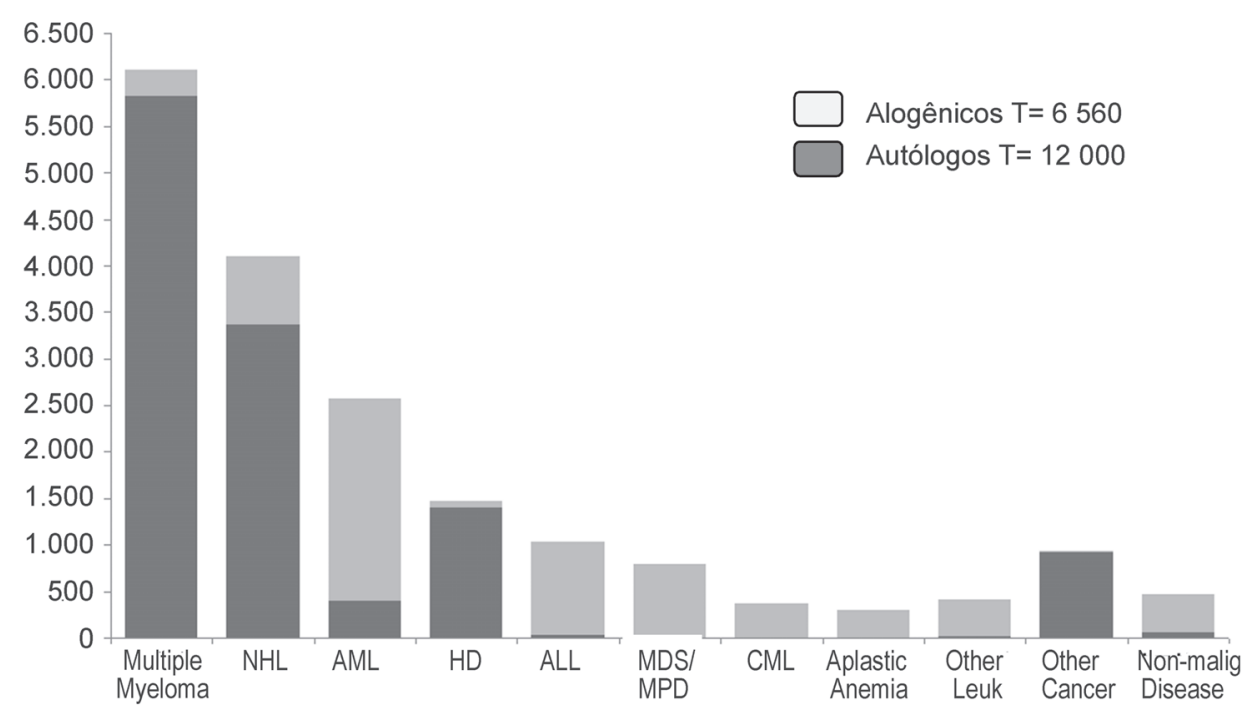

Figura 1. Indicações de transplante de células precursoras na America do Norte (2006). Fonte: Center for International Bone Marrow Transplantation Research; 2005

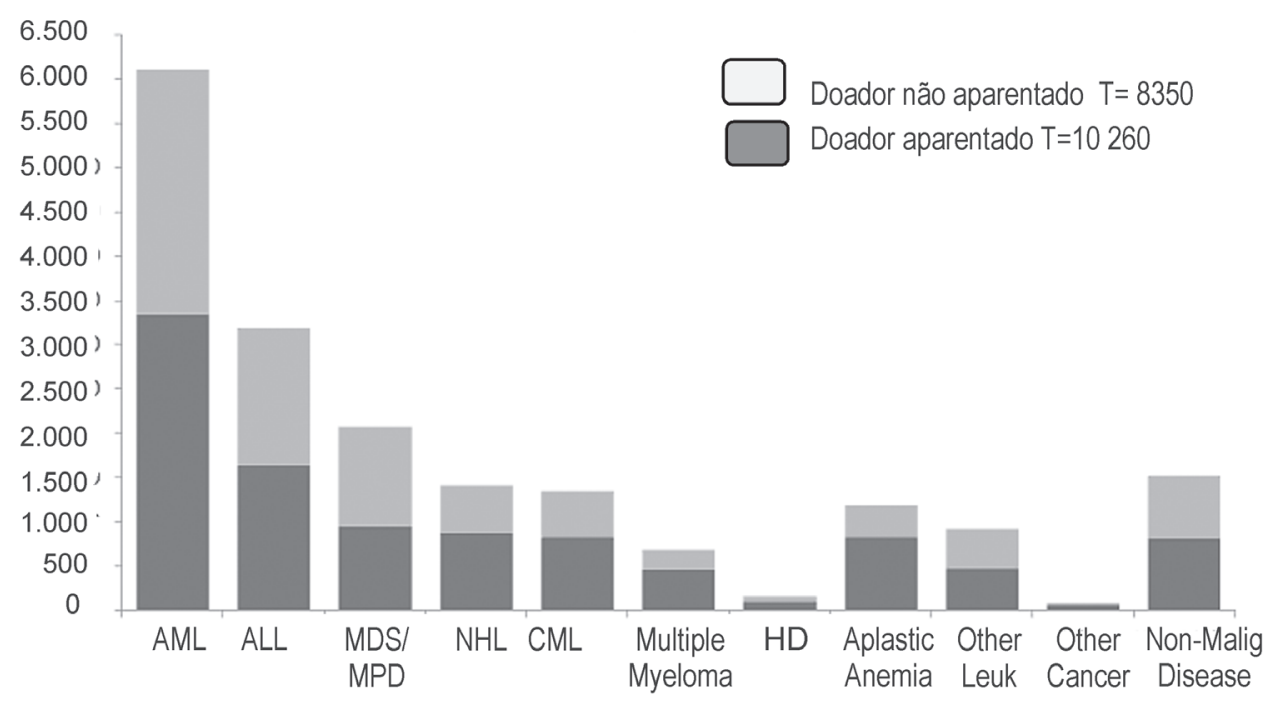

Figura 2. Indicações de transplante de células hematopoéticas mundial (2006). Fonte: Center for International Bone Marrow Transplantation Research; 2005 
frequência em pacientes em $1^{\mathrm{a}} \mathrm{RC}$ do que aqueles em $2^{\mathrm{a}} \mathrm{RC}$ ou mais. No registro europeu de transplantes, o número de transplantes em $1^{\mathrm{a}} \mathrm{RC}$ tornou-se maior em 2004 (Figura 3). No período entre 1998-2006, o CIBMTR, em publicação, revelou que $70 \%$ dos pacientes transplantados em remissão nos USA o foram em $1^{\mathrm{a}} \mathrm{RC}$.

$\mathrm{O}$ transplante em pacientes com LLA em $1^{\mathrm{a}} \mathrm{RC}$ revelam resultados de sobrevida estatisticamente superiores (Figuras 4 e 5, CIBMTR).

Storb et al, ${ }^{3}$ em uma avaliação de 182 pacientes adultos submetidos a transplante alogênico em diversos estádios da doença, revelaram que a sobrevida livre de eventos em cinco anos após o transplante foi de $43 \%$ para pacientes em $1^{\text {a }}$ $\mathrm{RC}, 23 \%$ para $2^{\mathrm{a}} \mathrm{RC}$ ou subsequentes remissões e apenas 9\% para pacientes em recidiva (Figura 6). O levantamento realizado em 16 instituições brasileiras (583 pacientes) demonstrou o mesmo valor quanto à sobrevida nos transplantes realizados em $1^{a}$ remissão. Zanichelli (comunicação pessoal). Figura 7

Estudos de metanálise demonstram vantagem, em pacientes de alto risco, com custo benefício favorável. ${ }^{4}$

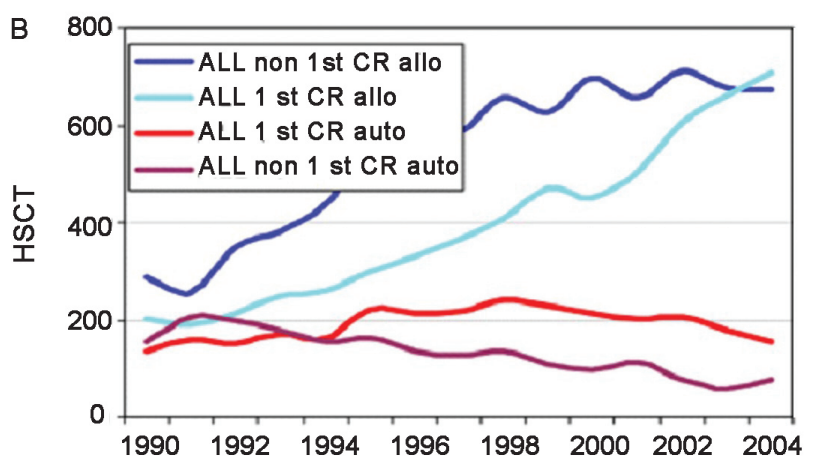

Figura 3. Registro Europeu - aumento progressivo da indicação de LLA em adultos em $1^{\text {a }}$ RCC

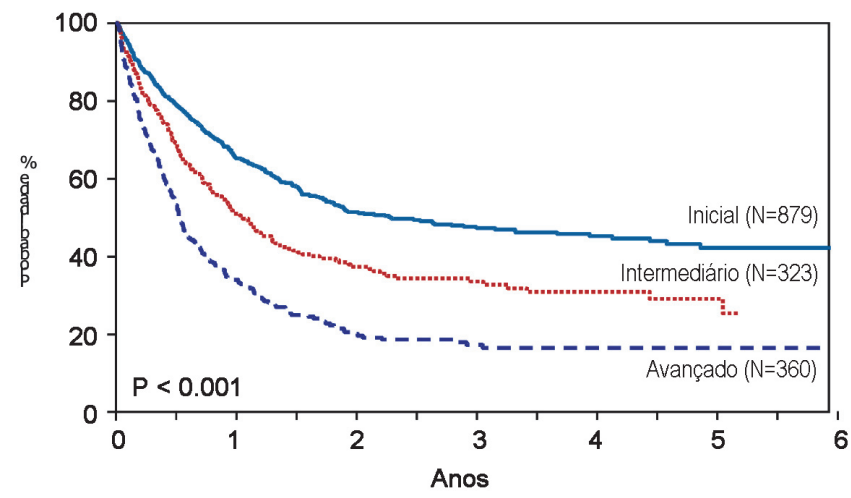

Figura 4. Probabilidade de sobrevida após transplante idêntico familiar para pacientes com LLA com idade $\geq 20$ anos de acordo com o estágio da doença (1998-2004). Fonte: Center for International Bone Marrow Transplantation Research; 2005

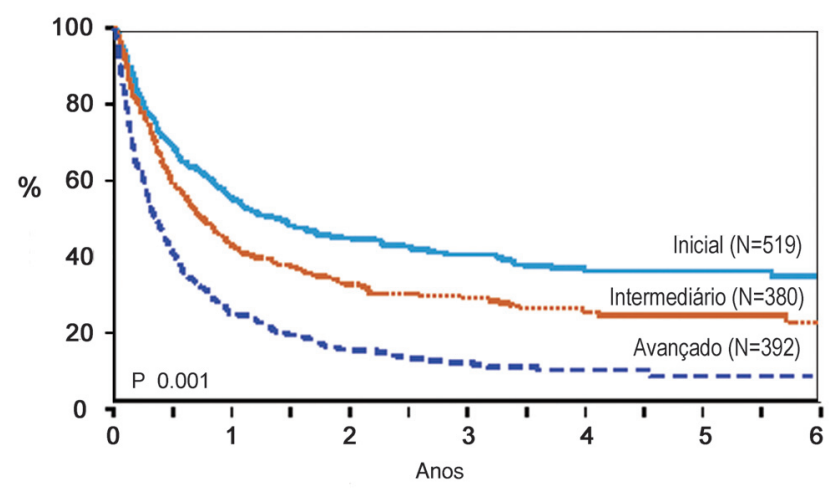

Figura 5. Probabilidade de sobrevida após transplante com doador não aparentado para LLA com idade > 20 anos (1998-2004). Fonte: Center for International Bone Marrow Transplantation Research; 2005

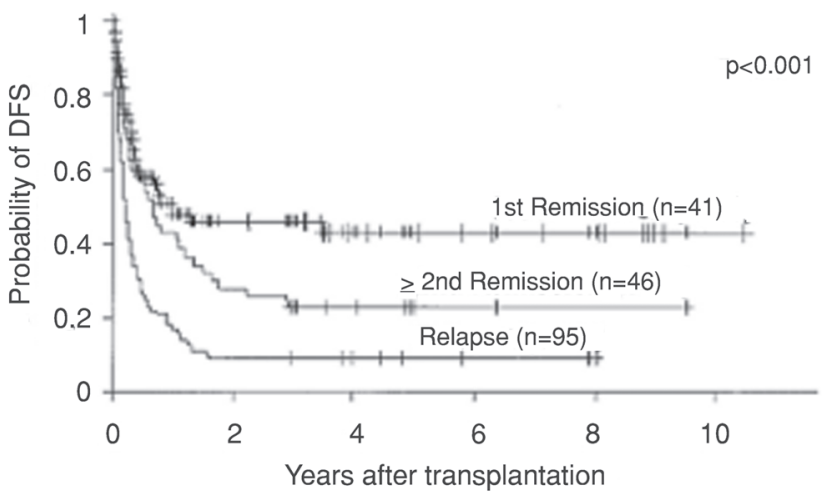

Figura 6. Probabilidade de doença livre de eventos em diversas situações pré-transplante alogênico de medula óssea

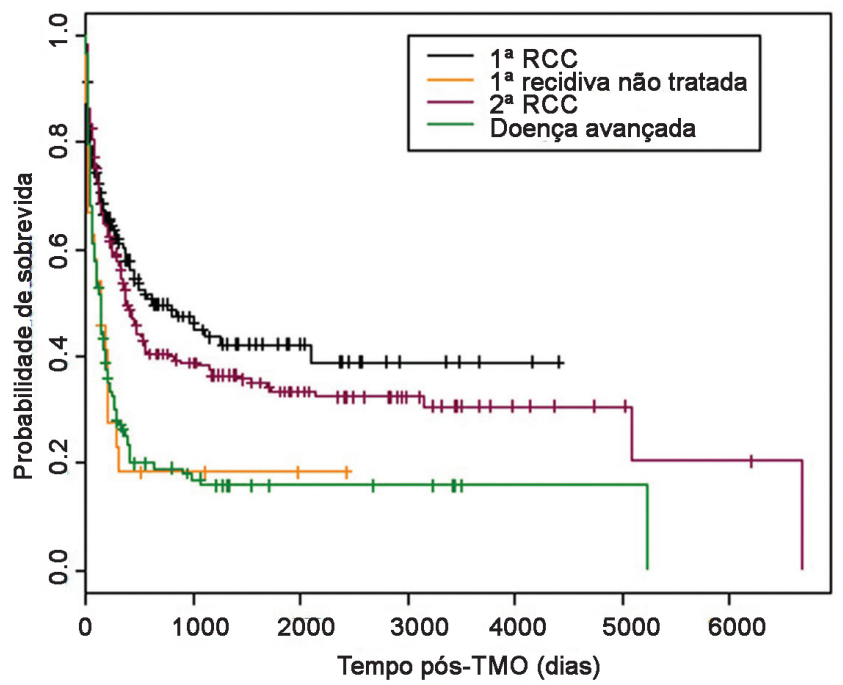

Figura 7. Sobrevida pós-TMO segundo situação pré-TMO experiência nacional 


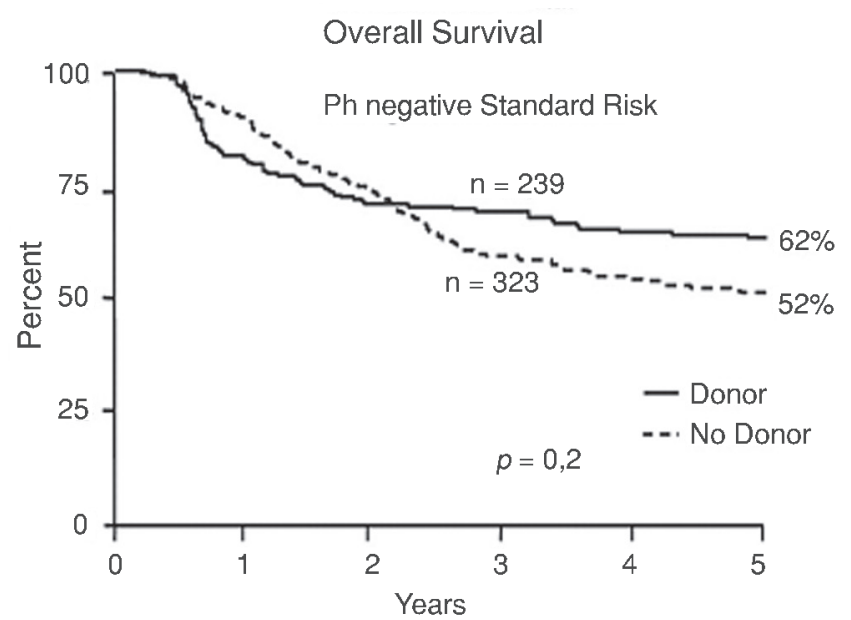

Figura 8. Sobrevida global de pacientes com LLA Ph negativo, baixo risco submetidos a TMO e quimioterapia convencional (sem doadores)

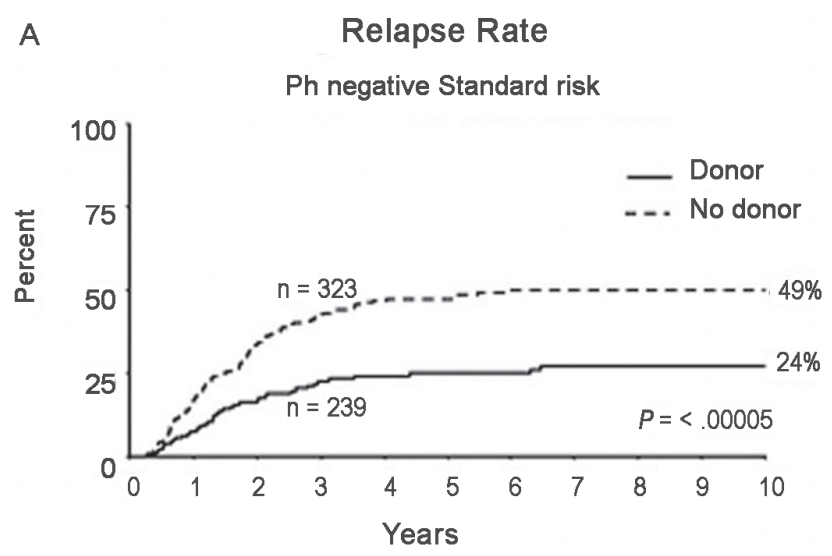

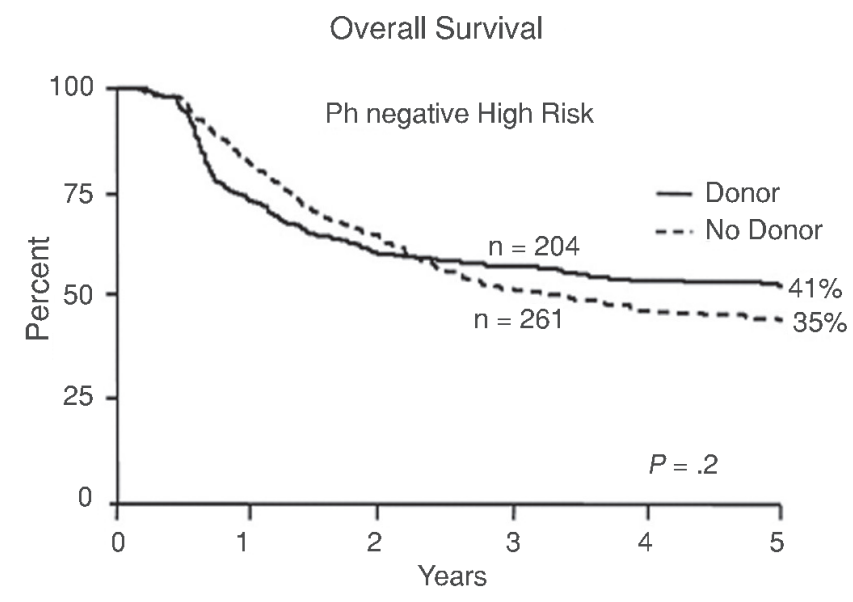

Figura 9. Sobrevida global de pacientes com de LLA Ph negativo, alto risco submetidos a TMO e quimioterapia convencional (sem doadores)

B

Relapse Rate

Ph negative High risk

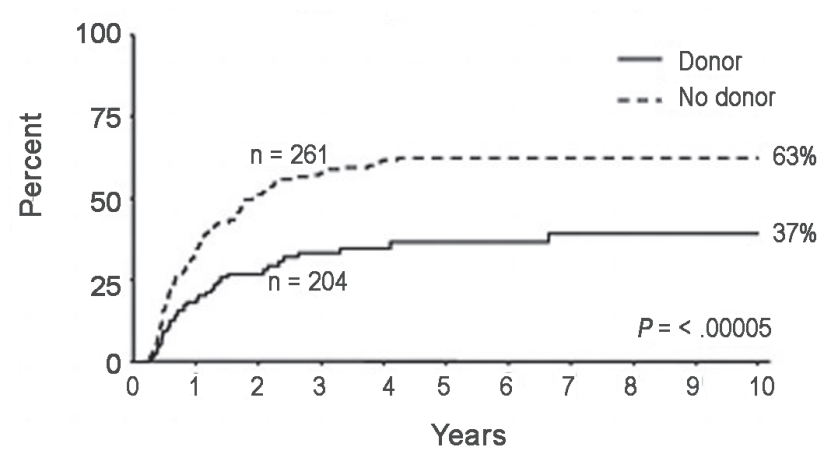

C Non-relapsed mortality (\%)

\begin{tabular}{|l|c|c|c|c|}
\hline & 3 months & 6 months & 1 year & 2 years \\
\hline High Risk & & & & \\
Donor & 1.5 & 7.3 & 26.0 & 35.8 \\
No Donor & 1.2 & 2.0 & 10.3 & 13.6 \\
\hline Standard Risk & & & & \\
$\quad$ Donor & 0.4 & 3.4 & 17.6 & 19.5 \\
No Donor & 0.3 & 1.2 & 5.3 & 6.9 \\
\hline
\end{tabular}

Figura 10. Taxas de recidiva. A) LLA Ph negativo, baixo risco; B) LLA Ph negativo, alto risco; C) taxas de mortalidade não relacionada a recidivas em pacientes submetidos a TMO e a quimioterapia. 
$\mathrm{O}$ transplante, quando realizado em $1^{\mathrm{a}} \mathrm{RC}$, apresenta menor taxa de recidiva e mortalidade relacionada ao transplante (MRT) do que em pacientes em $2^{\mathrm{a}} \mathrm{RC}$, respectivamente no TCTH aparentado e no TCTH não aparentado.

O grupo cooperativo MRC/ECOG publicou, em 2008 (Blood), os resultados comparando a evolução de pacientes adultos portadores de LLA, que dispunham de doadores familiares compatíveis, com aqueles que não dispunham de doadores, entre os últimos, comparando aqueles que realizaram TCTH autólogo, contra os que fizeram quimioterapia. Durante o período entre 1993 a 2006 foram incluídos 1.929 pacientes. Excluindo os pacientes com LLA Ph1+, os que não remitiram e aqueles que não foram testados para a disponibilidade de doadores familiares, restaram 1.150 pacientes. A análise de sobrevida global entre os pacientes de baixo risco foi significativamente melhor no grupo de pacientes que dispunha de doadores aparentados idênticos (Figura 8); já no grupo de alto risco, com disponibilidade de doadores, a sobrevida, embora melhor, não foi estatisticamente significante, do que aqueles que não dispunham de doador (Figura 9).

É interessante comentar que a alta taxa de MRT, verificada no grupo de alto risco, submetido a TCTH familiar idêntico, anulou o benefício de baixa recaída, diferentemente do que aconteceu com os pacientes do grupo de baixo risco, que também transplantaram (Figura 10).

O grupo que fez quimioterapia e não dispunha de doador familiar apresentou sobrevida estatisticamente melhor do que aqueles que realizaram TCTH autólogo, mostrando a falta de benefício dessa última opção (Figura 11).

Em publicação de 2009, Larson $^{5}$ conclui que o TCTH não deve ser feito de maneira indiscriminada para os pacientes adultos jovens, valorizando o resultado de quimioterapia intensiva nessa população, sugerindo que aqueles com baixo risco, que forem direcionados para a quimioterapia, sejam monitorados quanto à DRM, para possível redirecionamento de conduta. Goldstone, ${ }^{6}$ também em 2009, valorizou a indicação de TCTH em adultos em primeira

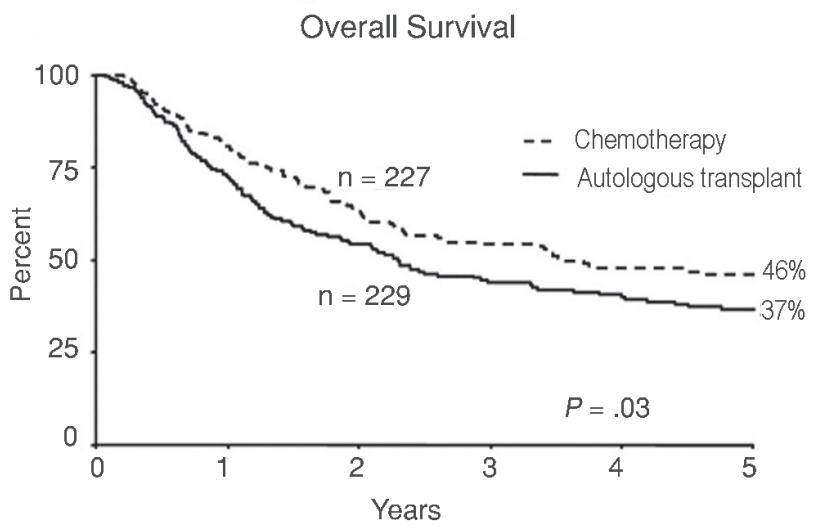

Figura 11. Sobrevida global entre transplante autólogo e quimioterapia não é significativa remissão, principalmente para aqueles com mais de 25 anos, uma vez que, na atualidade, existe a possibilidade de sobrevida livre de doença adequada para os pacientes adultos mais jovens, utilizando-se protocolos terapêuticos intensivos pediátricos

No grupo de alto risco, a indicação de TCTH está mantida, embora no resultado do MRC/ECOG, a melhor sobrevida verificada nos pacientes que dispunham de doador familiar não tenha atingido significância estatística, provavelmente devido a melhor sobrevida constatada nos estudos de metanálise (ref.) e diante de reconhecida menor recaída. Como a MRT na população maior que 35 anos é alta, estudos prospectivos controlados têm sido propostos, utilizando condicionamentos de intensidade reduzida, levando em conta os estimulantes resultados verificados previamente, ainda que não consolidados. ${ }^{7}$

\section{Transplante de célula-tronco hematopoética em leucemia linfoide aguda $\mathrm{Ph}+$}

Os pacientes com LLA $\mathrm{Ph}+$ têm sido transplantados rotineiramente com doadores familiares ou não aparentados, em primeira remissão. Em publicação de 2009, Fielding et al. ${ }^{8}$ relataram resultado da cooperação MRC/ECOG 2993 com melhor sobrevida livre de doença, para os pacientes adultos portadores de LLA que realizaram TCTH.

A utilização de inibidores de tirosina quinase em pacientes com LLA Ph+, associada à QT, está sendo cada vez mais valorizada.

Com a administração do imatinibe na dose de 400$600 \mathrm{mg}$ ficou demonstrado que houve aumento na taxa de RC ( $95 \%)$ e da negatividade de BCR/ABL (50\%) (MDACC, Pethema, JALSG, GMALL), não aumentou significativamente a toxicidade da quimioterapia (Pethema). Pode ser mantido após o TMO (GMALL) (Figura 12).

\section{Considerações}

- Pacientes adultos com LLA em $2^{\mathrm{a}} \mathrm{RC}$ têm chance limitada de cura com QT. TCTH está recomendado nessa situação.

- Atualmente, o resultado do TCTH não aparentado, compatível aproxima-se do aparentado idêntico, principalmente se considerarmos: Kiehl ${ }^{9}$

HLA de alta resolução Lee $^{10}$

Uso de globulina antitimócito ${ }^{11}$

- Diante da indisponibilidade de doador na família, dar preferência ao doador voluntário 8/8 ou 10/10 com HLA em alta resolução. Em casos emergenciais, considerar a utilização de sangue de cordão umbilical placentário (SCUP), incluindo a opção de duplo cordão, principalmente em adultos jovens. $^{12}$

- Dar preferência ao condicionamento com RCT, associado a ciclofosfamida ou etoposide. ${ }^{13}$ 

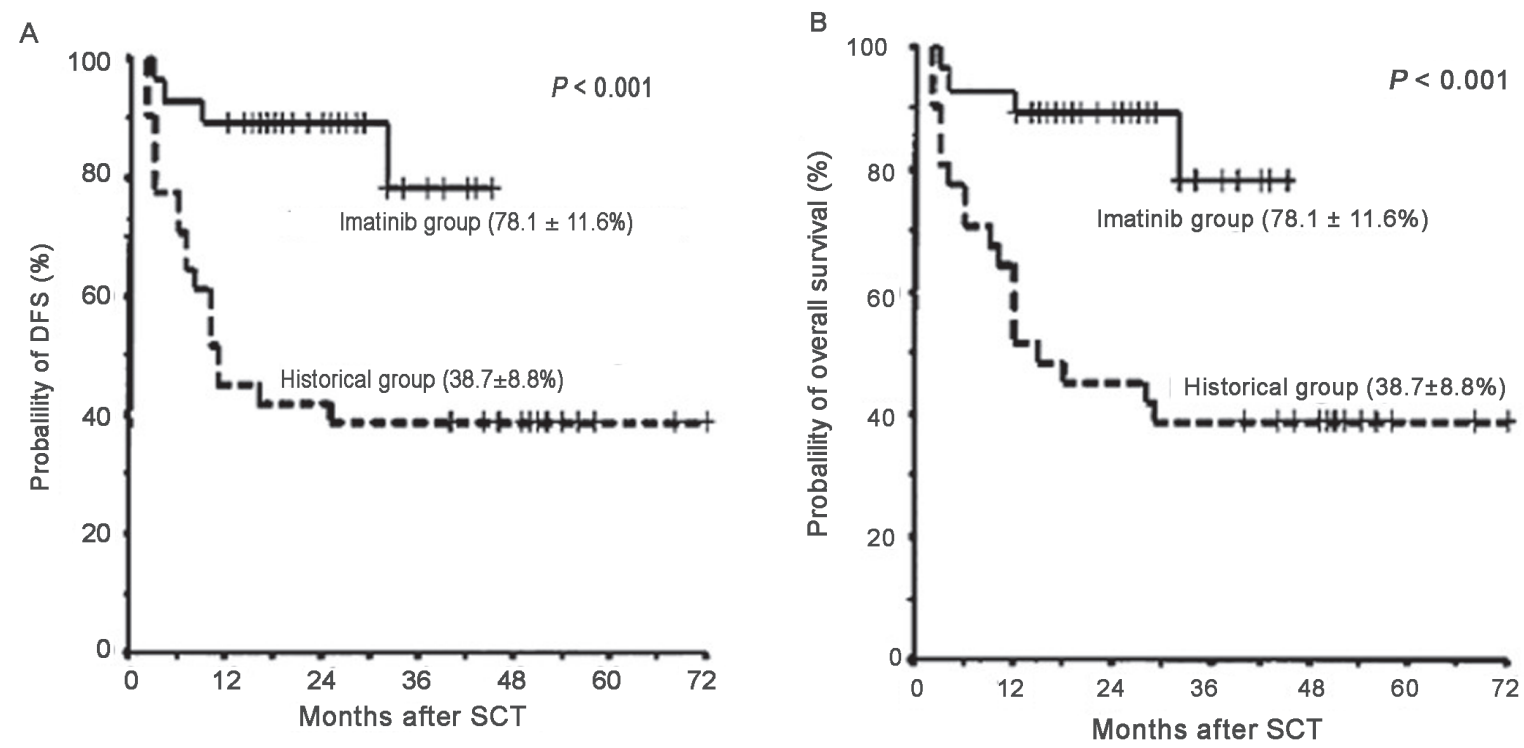

Figura 12. Sobrevida livre de eventos e sobrevida global pós TMO em pacientes LLA Ph positivo recebendo mesilato de imatinibe, comparado a grupo histórico

\section{Considerações finais}

- OTCTH tem sido amplamente utilizado em pacientes de alto risco, em $1^{\mathrm{a}} \mathrm{RC}$.

- Tem sua indicação valorizada em pacientes de baixo risco em $1^{\mathrm{a}} \mathrm{RC}$.

- Dificilmente teremos uniformidade em sua utilização, devendo ser uma opção presente para os pacientes com LLA em $1^{\mathrm{a}} \mathrm{RC}$

- Portaria 931, maio de 2006, contempla o TCTH em LLA $/ 1^{a} \mathrm{RC}$, se $\mathrm{Ph} 1+$, e deve ser adequada.

\section{Algoritmo de indicação de transplante de células-tronco hematopoéticas}

Pacientes com doador aparentado idêntico:

- LLA alto risco, $1^{\mathrm{a}} \mathrm{RC}$.

- LLA em $2^{\mathrm{a}} \mathrm{RC}$ ou mais.

- LA baixo risco, considerado e aceito, se indicado criteriosamente em pacientes com idade $>25$ e análise dos resultados obtidos com a quimioterapia anteriormente realizada.

Considerações

- Utilizar inibidor de tirosina quinase no pré-TCTH, nos pacientes com LLA $\mathrm{Ph}+$.

- Monitorizar DRM em pacientes jovens e baixo risco.

Pacientes com doador não aparentado compatível:

- LLA alto risco, $1^{\mathrm{a}} \mathrm{RC}$.

- LLA em $2^{\mathrm{a}} \mathrm{RC}$ ou mais.

\section{Considerações}

- Utilizar inibidor de tirosina quinase no pré-TCTH, nos pacientes com LLA $\mathrm{Ph}+$.

- Monitorizar DRM em pacientes jovens e baixo risco, em quimioterapia.

\begin{abstract}
In acute lymphoblastic leukemia, accumulation and proliferation of immature cells infiltration characterise a heterogeneous entity, featuring a wide variety of clinical and biological aspects. In the adult LLA concentration of high-risk prognosis factors such as age, $B$-cell, chromosomic changes, and chiefly the presence of chromosome positive Ph. Considerations of high morbidity and mortality rates related to haematopoietic stem cell transplantation (TCTH) have generated controversy about this therapeutic modality in adult patients with LLA in first remission (1st RC). The results of conventional therapy with chemotherapy in contrast with different risk groups of patients with LLA, has been used for the indication of TCTH. Thus we present the algorithm indications of haematopoietic stem cell transplantation in adult patients with LLA. Rev. Bras. Hematol. Hemoter. 2010;32(Supl. 1):54-60.
\end{abstract}

Key words: Acute lymphoblastic leukemia; adults; prognostics factors; haematopoietic stem cell transplantation.

\section{Referências Bibliográficas}

1. Thomas X, Boiron JM, Huguet F, Dombret H, Bradstock K, Vey N, et al. Outcome of treatment in adults with acute lymphoblastic leukemia: analysis of the LALA-94 trial. J Clin Oncol. 2004;22 (20):4075-86 
2. Nicola Gökbuget, Dieter Hoelzer. Treatment of adult acute lymphoblastic leukemia. Hematology Am Soc Hematol Educ Program. 2006:133-41.

3. Doney K, Hägglund H, Leisenring W, Chauncey T, Appelbaum FR, Storb R. Predictive factors for outcome of allogeneic hematopoietic cell transplantation for adult acute lymphoblastic leukemia. Biol Blood Marrow Transplant. 2003;9(7):472-81.

4. Orsi C, Bartolozzi B, Messori A, Bosi A. Event-free survival and cost-effectiveness in adult acute lymphoblastic leukaemia in first remission treated with allogeneic transplantation. Bone Marrow Transplant. 2007;40(7):643-9.

5. Larson RA. Allogeneic hematopoietic cell transplantation is not recommended for all adults with standard risk acute lymphoblastic leukemia in first complete remission". Biol Blood Marrow Transplant. 2009;1(suppl. 1):11-16.

6. Goldstone AH. Transplants in adult ALL-? Allo for everyone. Biol Blood Marrow Transplant. 2009;1(suppl. 1):7-10.

7. Stein A, Forman SJ. Allogeneic transplantation for ALL in adults. Bone Marrow Transplant. 2008;41(5):439-46.

8. Fielding AK, AH Goldstone AH. Allogeneic haematopoietic stem cell transplant in Philadelphia-positive acute lymphoblastic leukaemia. Bone Marrow Transplantation of long term survivors: report from Bone Marrow Trasplant-Survivors Study. Blood. 2008; 110(41):3447-53.

9. Kiehl MG, Kraut L, Schwerdtfeger R, Hertenstein B, Remberger $\mathrm{M}$, Kroeger N, et al. Outcome of allogeneic hematopoietic stemcell transplantation in adult patients with acute lymphoblastic leukemia: no difference in related compared with unrelated transplant in first complete remission. J Clin Oncolo. 2004; 22(14):2816-25.

10. Lee SJ, Klein J, Haagenson M, Baxter-Lowe LA, Confer DL, Eapen M, et al. High-resolution for-recipient HLA matching contributes to the success of unrelated donor marrow transplantation. Blood. 2007;110(13):4576-83.

11. Kröger N, et al. HLA-mismatched unrelated donors as an alternative graft source for allogeneic stem cell transplantation after antithymocyte globulin-containing conditioning regimen. Biol Blood Marrow Transplant. 2009;15(4):454-62

12. Bachanova V, Bachanova V, Verneris MR, DeFor T, Brunstein CG, Weisdorf DJ. Prolonged survival in adults with acute lymphoblastic leukemia after reduced-intensity conditioning with cord blood or sibling donor transplantation. Blood. 2009; 113(13):2902-5.

13. Marks DI, Aversa F, Lazarus HM. Alternative donor transplants for adult acute lymphoblastic leukaemia: a comparison of the three major options. Bone Marrow Transplant. 2006;38(7):467-75.

Este artigo foi avaliado pelos coordenadores das Diretrizes do Transplante de Medula Óssea da Sociedade Brasileira de Transplante de Medula Óssea, Luis Fernando Bouzas, Prof. Julio Cesar Voltarelli e Nelson Hamerschlak, e publicado após avaliação e revisão do editor, Milton Artur Ruiz.

Conflito de interesse: sem conflito de interesse

Recebido: 05/11/2009

Aceito: 23/11/2009 